\title{
Cohomologies of Lie Algebras of Vector Fields with Coefficients in Adjoint Representations Hamiltonian Case
}

\author{
By \\ Yukihiro KANIE*
}

\section{Introduction}

Let $M$ be a smooth manifold, and $\operatorname{sr}(M)$ the infinite dimensional Lie algebra of all smooth vector fields on $M$. Let $\mathfrak{A}$ be $\mathfrak{I}(M)$ or a certain natural subalgebra of it. We arc interested in the cohomology $H^{*}(\mathfrak{I} ; V)$ of $2 \mathbb{f}$ with coefficients in some representation $V$, which is an invariant of the Lie algebra $\mathfrak{N}$.

In 1968, I. M. Gel'fand and D. B. Fuks began to study the theory of cohomologies of Lie algebras of vector fields. First, they treated the case where $\mathfrak{H}=\mathfrak{H}(M)$ and $V=\mathbb{R}$ (trivial coefficients). Since then, many mathematicians studied cohomologies in many cases, for instance [2], [4], [6] etc. They also treated the case of nontrivial coefficients, but restricted themselves to the representations induced from some finite dimensional ones. Their proofs were essentially based upon some finiteness of representations.

Meanwhile, in 1973, F. Takens [7] proved that any derivations of $\mathfrak{A}(M)$ is inner. It means that the first cohomology of $\mathfrak{A}(M)$ with coefficients in its adjoint representation, a natural infinite dimensional representation, is trivial.

In the present papcr, the author treats a symplectic manifold $(M, \omega)$ and the subalgebra $\mathfrak{A}_{o}(M)$ consisting of hamiltonian vector fields on $M$ in this direction. Then he obtains the following results.

Main Theorem. Let $(M, \omega)$ be a connected symplectic manifold,

Communicated by H. Yoshizawa, August 5, 1974. Revised September 2, 1974.

* Graduate School, Kyoto University, Kyoto. 
then the first cohomology of $\mathfrak{H}_{\omega}(M)$ with coefficients in its adjoint representation, is of dimension 1 or 0 , that is,

$$
\operatorname{dim} H^{1}\left(\mathfrak{U}_{\omega}(M) ; \mathfrak{I}_{\omega}(M)\right)=1 \text { or } 0
$$

Moreover, $H^{1}\left(\mathfrak{U}_{\omega}(M) ; \mathfrak{A}_{\omega}(M)\right) \cong \mathbf{R}$ if and only if the symplectic form $\omega$ is exact.

Locally, this theorem has a simple feature (Theorem 5) as follows: Let $U$ be a connected and simply connected open set in $\mathbb{R}^{2 n}$, with the natural symplectic structure $\omega=\Sigma d x_{i} d y_{i}$, then

$$
H^{1}\left(\mathfrak{U}_{\omega}(U) ; \mathfrak{H}_{\omega}(U)\right) \cong \mathbf{R} .
$$

The proof of Main Theorem can be carried out by elementary calculations. But to make short some part of the proof, we use Weyl's results on representations of the symplectic algebra. The elementary version of that part is outlined also in Section 4.

In $\S 1$, we explain some generalities of the first cohomology and symplectic manifolds. In $\S 2$, we prove interesting properties (Propositions 1 and 4) of hamiltonian vector fields, which play an important role to prove Theorem 5, a local theorem. Moreover we prove in $\S 2$ that a derivation of $\mathfrak{A}_{\omega}(M)$ is a local operator (Proposition 3). Section 3 is devoted to the study of derivations of $\mathfrak{U}_{\omega}(M)$ in local. In $\S 4$, we complete the proof of Theorem 5. Here we use some knowledge of formal hamiltonian vector fields. Finally in $\S 5$, we give the proof of Main Theorem.

The author expresses his hearty thanks to Professors T. Hirai, T. Morimoto and N. Tatsuuma for their kind advices.

\section{§1. Derivations and $\boldsymbol{H}^{1}(\mathfrak{U} ; \mathfrak{U})$}

All manifolds, vector fields, functions etc. are assumed to be of $C^{\infty}$-class.

Let $\mathfrak{A}$ be a subalgebra of the Lie algebra $\mathfrak{U}(M)$ of all vector fields on a manifold $M$, and consider the adjoint representation of $\mathfrak{A}$ :

$$
(\operatorname{ad} X)(Y)=[X, Y] \quad(X, Y \in \mathfrak{A}),
$$


where $[$,$] is the usual bracket operation of vector fields. The co-$ chain complex $\left\{C^{q}(\mathfrak{U} ; \mathfrak{U}), d^{q}\right\}$ of the Lie algebra $\mathfrak{U}$ with coefficients in its adjoint representation consists of the following:

$C^{q}(\mathfrak{A} ; \mathfrak{U})=\{P: \mathfrak{A} \times \cdots \times \mathfrak{U} \longrightarrow \mathfrak{A}$, skew-symmetric $q$-linear map $\}$, and for $P \in C^{q}$ and $X_{1}, \ldots, X_{q+1} \in \mathfrak{A}$,

$$
\begin{aligned}
\left(d^{q} P\right)\left(X_{1}, \ldots, X_{q+1}\right)= & \sum_{i=1}^{q+1}(-1)^{i+1}\left[X_{i}, P\left(X_{1}, \ldots, \hat{X}_{i}, \ldots, X_{q+1}\right)\right] \\
& +\sum_{i<j}(-1)^{i+j} P\left(\left[X_{i}, X_{j}\right], X_{1}, \ldots, \hat{X}_{i}, \ldots, \hat{X}_{j}, \ldots, X_{q+1}\right) .
\end{aligned}
$$

The homologies $\left\{H^{q}(\mathfrak{A} ; \mathfrak{A}), q \geqq 0\right\}$ of this complex are called the cohomologies of $\mathfrak{A}$ with coefficients in its adjoint representation. The one dimensional cohomology $H^{1}(\mathfrak{A} ; \mathfrak{A})$ is interpreted as follows. Since

$$
d P(X, Y)=[X, P(Y)]-[Y, P(X)]-P([X, Y]) \quad(X, Y \in \mathfrak{A}),
$$

for $P \in C^{1}$, we see that $d P=0$ means that

$$
P([X, Y])=[P(X), Y]+[X, P(Y)],
$$

that is, 1-dimensional cocycles are derivations of $\mathfrak{A}$. Moreover since

$$
(d Q)(X)=[X, Q] \quad(X \in \mathfrak{H}),
$$

for $Q \in C^{0}(\mathfrak{A} ; \mathfrak{N})=\mathfrak{A}$, we see that 1 -dimensional coboundaries are inner derivations of $\mathfrak{U}$. Thus the first cohomology space $H^{1}(\mathfrak{A} ; \mathfrak{A})$ is the equivalence classes of the algebra $\mathfrak{D}(\mathfrak{A})$ of derivations of $\mathfrak{A}$ modulo its ideal $\mathfrak{D}^{i}(\mathfrak{U})$ of inner derivations, or

$$
H^{1}(\mathfrak{U} ; \mathfrak{A}) \cong \mathfrak{D}(\mathfrak{U}) / \mathfrak{D}^{i}(\mathfrak{U})
$$

In the following, we consider a smooth symplectic manifold $\left(M^{2 n}, \omega\right)$, and the subalgebra $\mathfrak{A}=\mathfrak{A}_{\omega}(M)$ of hamiltonian vector fields on $M$. A symplectic structure is defined on $M^{2 n}$ by a nondegenerate closed 2-form $\omega$, that is, $\omega^{n}=\omega \wedge \cdots \wedge \omega$ is a volume form of $M$ and $d \omega=0$. A vector field $X$ is called hamiltonian, if it preserves the symplectic form $\omega$, and by definition,

$$
\mathfrak{A}_{\omega}(M)=\left\{X \in \mathfrak{A}(M) ; L_{X} \omega=0\right\},
$$


where $L_{X} \omega$ is the Lie derivative of $\omega$ by $X$. To determine the first cohomology, we must study the structures of $\mathfrak{D}_{\omega}(M)=\mathfrak{D}\left(\mathfrak{A}_{\omega}(M)\right)$ and $\mathfrak{D}_{\omega}^{i}(M)=\mathfrak{D}^{i}\left(\mathfrak{H}_{\omega}(M)\right)$.

\section{§2. Some Properties of $\mathfrak{V}_{\omega}(\mathbb{M})$ and $D_{\omega}(\mathbb{M})$}

2.1. In the following, we denote by $\partial_{v}$ the vector field $\frac{\partial}{\partial v}$, and by $X_{\mid U}$ the restriction of $X$ on $U$.

Proposition 1. Let $p$ be a point of a symplectic manifold $M^{2 n}$, and let $X$ be a hamiltonian vector field on $M$ such that $j^{2}(X)(p)=0$, that is, the 2-jet of each of the coefficient functions of $X$ is zero at $p$. Then, there exist a finite number of hamiltonian vector fields $Y_{1}, \ldots$, $Y_{l}, Z_{1}, \ldots, Z_{l}$ on $M$, and a neighbourhood $U$ of $p$ in $M$, such that

$$
X_{\mid U}=\sum_{i=1}^{l}\left[Y_{i}, Z_{i}\right]_{\mid U}
$$

and

$$
j^{1}\left(Y_{i}\right)(p)=j^{1}\left(Z_{i}\right)(p)=0 \quad(1 \leqq i \leqq l)
$$

Proof. Let $U$ be a simply connected open neighbourhood of $p$, and let $\left(x_{1}, \ldots, x_{n}, y_{1}, \ldots, y_{n}\right)$ be a symplectic coordinate system around $p$ in $U$, that is,

$$
\omega_{\mid U}=\sum_{i=1}^{n} d x_{i} d y_{i}
$$

Since the vector field $X$ is hamiltonian, $L_{X} \omega=d i_{X} \omega=0$, where $i_{X} \omega$ is the interior product of $X$ and $\omega$. Hence the differential form $i_{X} \omega$ is closed, and so the restriction $i_{X} \omega_{\mid U}$ is exact by Poincaré's lemma. With respect to the above local coordinates, $X$ and $i_{X} \omega$ are written in $U$ as

$$
\begin{gathered}
X=\sum_{i=1}^{n}\left\{f_{i}(x, y) \partial_{x_{i}}+g_{i}(x, y) \partial_{y_{i}}\right\}, \\
i_{X} \omega=\sum_{i=1}^{n}\left\{f_{i}(x, y) d y_{i}-g_{i}(x, y) d x_{i}\right\},
\end{gathered}
$$

where $f_{\imath}$ and $g_{i}$ are functions on $U$. There exists a function $H=H(x, y)$ 
on $U$ such that

$$
i_{X} \omega=d H=\sum_{i=1}^{n}\left(H_{x_{i}} d x_{i}+H_{y_{i}} d y_{i}\right)
$$

Therefore we have for $i=1, \ldots, n$,

$$
f_{i}(x, y)=H_{y_{i}}, \quad g_{i}(x, y)=-H_{x_{i}},
$$

that is,

$$
X=\sum_{i=1}^{n}\left(H_{y_{i}} \partial_{x_{i}}-H_{x_{i}} \partial_{y_{i}}\right) \quad \text { on } U
$$

This function $H$ is uniquely determined up to constants, so that we may put $H(p)=0$. A function or vector field is called without constant term if it is zero at the origin of the coordinate system.

In global, any function $H$ on $M$ uniquely determines the hamiltonian vector field $X$ on $M$ by the formula $i_{X} \omega=d H$, because of nondegeneracy of the symplectic form $\omega$. So $X$ may be written as $X_{H}$. Then the following formula holds for two functions $H$ and $K$ on $M$,

$$
\left[X_{H}, X_{K}\right]=X_{-\{H, K\}} \cdot
$$

Here $\{H, K\}$ is a function on $M$ called the Poisson bracket of $H$ and $K$, which is given in $U$ by local coordinates as

$$
\{H, K\}=\sum_{i=1}^{n}\left(H_{x_{i}} K_{y_{i}}-H_{y_{i}} K_{x_{i}}\right)
$$

Thus, the proposition follows from the following result on a connected open set (called domain) in a Euclidean space.

Q.E.D.

Proposition 2. Let $H$ be a $C^{\infty}$-function on a simply connected domain $U$ in $R^{2 n}$ with $j^{3}(H)(0)=0$, then there exist a finite number of $C^{\infty}$-functions $K_{1}, \ldots, K_{l}, G_{1}, \ldots, G_{l}$ on $U$, such that

$$
H=\sum_{i=1}^{l}\left\{K_{i}, G_{i}\right\}
$$

and

$$
j^{2}\left(K_{i}\right)(0)=j^{2}\left(G_{i}\right)(0)=0 \quad(1 \leqq i \leqq l)
$$


Proof. Since $j^{3}(H)(0)=0, H$ can be given as a finite sum of functions of the following form:

$$
x_{1}^{l_{1}} \ldots x_{n}^{l_{n}} y_{1}^{m_{1}} \ldots y_{n}^{m_{n}} f(x, y)
$$

with $\sum_{i=1}^{n}\left(l_{i}+m_{i}\right) \geqq 4$, and $f$ a $C^{\infty}$-function on $U$. Since $\sum_{i=1}^{n} l_{i} \geqq 2$, or $\sum_{i=1}^{n} m_{i} \geqq 2$, we may assume $\sum_{i=1}^{n} l_{i} \geqq 2$ without loss of generality.

CASE 1. The case where $l_{i_{0}} \geqq 2$ for some $i_{0}$. Let $i_{0}=1$, and put

$$
K=x_{1}^{3}, G=x_{1}^{l_{1}-2} \prod_{i=2}^{n} x_{i}^{l_{i}} y_{i}^{m_{i}} g(x, y)
$$

where

$$
g=\frac{1}{3} \int_{0}^{y_{1}} y_{1}^{m_{1}} f(x, y) d y_{1},
$$

then we have

$$
\begin{aligned}
\{K, G\} & =3 x_{1}^{l_{1}} \prod_{i=2}^{n} x_{i}^{l_{i}} y_{i}^{m_{i}} g_{y_{1}}(x, y) \\
& =x_{1}^{l_{1}} \ldots x_{n}^{l_{n}} y_{1}^{m_{1}} \ldots y_{n}^{m_{n}} f(x, y) .
\end{aligned}
$$

Moreover $j^{2}(K)(0)=j^{2}(G)(0)=0$, because $j^{m_{1}}(g)(0)=0$ and

$$
\sum_{i=2}^{n}\left(l_{i}+m_{i}\right)+\left(l_{1}-2\right)+\left(m_{1}+1\right)=\sum_{i=1}^{n}\left(l_{i}+m_{i}\right)-1 \geqq 3 \text {. }
$$

CASE 2. The case where all $l_{i} \leqq 1$. Assume that $l_{1}=l_{2}=1$, then by means of the following symplectic transformation, this case is reduced to Case 1:

$$
\begin{cases}x_{1}^{\prime}=\sqrt{2}^{-1}\left(x_{1}+x_{2}\right), & y_{1}^{\prime}=\sqrt{2}^{-1}\left(y_{1}+y_{2}\right), \\ x_{2}^{\prime}=\sqrt{2}^{-1}\left(x_{1}-x_{2}\right), & y_{2}^{\prime}=\sqrt{2}^{-1}\left(y_{1}-y_{2}\right), \\ x_{i}^{\prime}=x_{i}, & y_{i}^{\prime}=y_{i} \quad(i \geqq 3) .\end{cases}
$$

Q.E.D. 
2.2. Proposition 3. Let $D$ be a derivation of $\mathfrak{A}_{\omega}(M)$. If $X \in \mathfrak{A}_{\omega}(M)$ is identically zero on some domain $U$ in $M$, then $D(X)$ vanishes identically on $U$.

Proof. Assume that there exists a point $p$ in $U$ such that $D(x)(p) \neq$ 0 . Let $V$ be a simply connected coordinate neighbourhood of $p$ in $U$. Since $D(X)$ is hamiltonian, using symplectic coordinates around $p$ in $V$, we can find a function $H$ on $V$ such that $D(X)_{\mid V}=X_{H}$, as in the proof of Proposition 1. Since $D(X)(p) \neq 0, H_{x_{i}}(p) \neq 0$ or $H_{y_{i}}(p) \neq 0$ for some $i$. We may assume that $H_{x_{i}}(p) \neq 0$. Let $K$ be a function whose support is contained in $V$, and equals to $y_{1}^{2}$ in a smaller neighbourhood $V^{\prime}$ of $p$. Then we have

$$
\{H, K\}=2 y_{i} H_{x_{i}} \quad \text { in } \quad V^{\prime},
$$

and then

$$
\{H, K\}_{y_{i}}=2 H_{x_{i}}+2 y_{i} H_{x_{i} y_{i}} .
$$

Hence

$$
X_{-\{H . K\}}(p)=-H_{x_{i}}(p) \partial_{x_{i}} \neq 0 .
$$

On the other hand, since $\left[X, X_{K}\right]=0$ on $M$,

$$
\begin{aligned}
0=D\left(\left[X, X_{K}\right]\right)(p) & =\left[D(X), X_{K}\right](p)+\left[X, D\left(X_{K}\right)\right](p) \\
& =X_{-\{H, K\}}(p) .
\end{aligned}
$$

This contradicts our assumption.

Q.E.D.

Proposition 4. Let $D$ be a derivation of $\mathfrak{H}_{\omega}(M)$, and $X$ be a hamiltonian vector field on $M$. If $j^{2}(X)(p)=0$ for some point $p$ of $M$, then $D(X)(p)=0$.

Proof. We can find, by Proposition 1, a neighbourhood $U$ of $p$, and hamiltonian vector fields $Y_{1}, \ldots, Y_{l}$ and $Z_{1}, \ldots, Z_{l} \in \mathfrak{A}_{\omega}(M)$ such that

$$
\begin{aligned}
& X_{\mid U}=\sum_{i=1}\left[Y_{i}, Z_{i}\right]_{\mid U}, \\
& j^{1}\left(Y_{i}\right)(p)=j^{1}\left(Z_{i}\right)(p)=0 \quad(1 \leqq i \leqq l) .
\end{aligned}
$$


Then, using Proposition 3, we get

$$
\begin{aligned}
D(X)(p) & =D\left(\sum_{i}\left[Y_{i}, Z_{i}\right]\right)(p) \\
& =\sum_{i}\left(\left[D\left(Y_{i}\right), Z_{i}\right](p)+\left[Y_{i}, D\left(Z_{i}\right)\right](p)\right)=0 .
\end{aligned}
$$

Q.E.D.

Remark 1. Any derivation $D$ of $\mathfrak{A}_{\omega}(M)$ can be considered as a derivation of $\mathfrak{A}_{\omega}(U)$ for any open subset $U$ of $M$.

In fact, for any point $p$ in $U$, by the proof of Proposition 1 , we have a hamiltonian vector field $\tilde{X}$ on $M$ for any $X \in \mathfrak{A}_{\omega}(U)$ such that $\tilde{X}$ equals to $X$ on some neighbourhood of $p$. Define $D_{U}$ by $D_{U}(X)(p)=$ $D(\tilde{X})(p)$, then $D_{U}(X)(p)$ is well defined by Proposition 3 , and clearly $D_{U}$ is a derivation of $\mathfrak{H}_{\omega}(U)$.

\section{§3. Inner Derivations of $\mathfrak{U}_{\omega}(\boldsymbol{U})$}

3.1. In this section, we fix a simply connected domain $U$ of $M$, and a coordinate system $\left(x_{1}, \ldots, x_{n}, y_{1}, \ldots, y_{n}\right)$ in $U$ for which $\omega=\Sigma d x_{i} d y_{\imath}$ in $U$. The conditions that a vector field $X$ on $U$ is hamiltonian, is given as follows:

$$
\begin{gathered}
X=\sum_{i=1}^{n}\left(f_{i}(x, y) \partial_{x_{i}}+g_{i}(x, y) \partial_{y_{i}}\right) \in \mathfrak{Q}_{\omega}(U) \\
\Leftrightarrow \partial_{x_{j}}\left(f_{i}\right)=-\partial_{y_{i}}\left(g_{j}\right), \partial_{y_{j}}\left(f_{i}\right)=\partial_{y_{i}}\left(f_{j}\right), \partial_{x_{j}}\left(g_{i}\right)=\partial_{x_{i}}\left(g_{j}\right) \\
(1 \leqq i, j \leqq n) .
\end{gathered}
$$

Theorem 5. Let $D$ be a derivation of the Lie algebra $\mathfrak{A}_{\omega}(U)$ of hamiltonian vector fields on $U$.

(i) There exists a unique vector field $Z$ (not necessarily hamiltonian) on $U$ such that

$$
D(X)=[Z, X] \quad\left(X \in \mathfrak{A}_{\omega}(U)\right) .
$$

(ii) $Z$ is uniquely expressed as $Z=Z_{1}+Z_{2}$, where $Z_{1} \in \mathfrak{A}_{\omega}(U)$ and for some constant $c$, 


$$
Z_{2}=c \sum_{i=1}^{n}\left(x_{i} \partial_{x_{i}}+y_{i} \partial_{y_{i}}\right)
$$

Note. If $c \neq 0, Z_{2}$ is not hamiltonian, because $L_{Z_{2}} \omega=2 c \omega$ in $U$.

Let us call a vector field constant or linear if it has only constant coefficients or linear coefficients respectively.

We construct the vector field $Z$ as a sum of $Z^{(0)}, Z^{(1)}$ and $Z^{(2)}$ :

$$
Z=Z^{(0)}+Z^{(1)}+Z^{(2)}
$$

Here $Z^{(0)}$ is the constant term of $Z$, and $Z^{(1)}$ is the linear term of $Z$ (containing $Z_{2}$ ), and finally $Z^{(2)}$ is the remaining term with coefficient functions of degree $\geqq 2$, a hamiltonian field.

\subsection{Determination of $Z^{(2)}$}

According to the situation, $x_{i}$ is denoted by $v_{i}$, and $y_{i}$ by $v_{i+n}$ for $1 \leqq i \leqq n$. To determine $Z^{\prime}=Z^{(1)}+Z^{(2)}$, we will use the following equalities,

$$
D\left(\partial_{v_{\imath}}\right)=\left[Z, \partial_{v_{\imath}}\right]=\left[Z^{\prime}, \partial_{v_{i}}\right] \quad(1 \leqq i \leqq 2 n)
$$

Define for all $i$ and $j$, the functions $f_{i j}$ etc. on $U$ as

$$
\begin{aligned}
& D\left(\partial_{\lambda_{i}}\right)=\sum_{j=1}^{n}\left(f_{i j} \partial_{x_{j}}+g_{i j} \partial_{y_{j}}\right), \\
& D\left(\partial_{y_{t}}\right)=\sum_{j=1}^{n}\left(f_{i j}^{\prime} \partial_{x_{j}}+g_{i j}^{\prime} \partial_{y_{j}}\right) .
\end{aligned}
$$

It follows from $\left[\partial_{v_{1}}, \partial_{v_{m}}\right]=0$ for $l, m=1, \ldots, 2 n$ that

$$
\begin{aligned}
0 & =D\left(\left[\partial_{x_{l}}, \partial_{x_{m}}\right]\right)=\left[D\left(\partial_{x_{l}}\right), \partial_{x_{m}}\right]+\left[\partial_{x_{l}}, D\left(\partial_{x_{m}}\right)\right] \\
& =\sum_{j}\left\{\left(\partial_{x_{l}}\left(f_{m j}\right)-\partial_{x_{m}}\left(f_{l j}\right)\right) \partial_{x_{j}}+\left(\partial_{x_{l}}\left(g_{m j}\right)-\partial_{x_{m}}\left(g_{l j}\right)\right) \partial_{y_{j}}\right\}, \\
0 & =D\left(\left[\partial_{x_{l}}, \partial_{y_{m}}\right]\right) \\
& =\sum_{j}\left\{\left(\partial_{x_{l}}\left(f_{m j}^{\prime}\right)-\hat{\partial}_{y_{m}}\left(f_{l j}\right)\right) \partial_{x_{j}}+\left(\partial_{x_{l}}\left(g_{m j}^{\prime}\right)-\partial_{y_{m}}\left(g_{l j}\right)\right) \partial_{y_{j}}\right\},
\end{aligned}
$$

and that 


$$
\begin{aligned}
0 & =D\left(\left[\partial_{y_{l}}, \partial_{y_{m}}\right]\right) \\
& =\sum_{j}\left\{\left(\partial_{y_{l}}\left(f_{m j}^{\prime}\right)-\partial_{y_{m}}\left(f_{l_{j}}^{\prime}\right)\right) \partial_{x_{j}}+\left(\partial_{y_{l}}\left(g_{m j}^{\prime}\right)-\partial_{y_{m}}\left(g_{l j}^{\prime}\right)\right) \partial_{y_{j}}\right\} .
\end{aligned}
$$

Therefore we have for all $j, l, m$,

$$
\begin{aligned}
& \partial_{x_{l}}\left(f_{m j}\right)=\partial_{x_{m}}\left(f_{l j}\right), \quad \partial_{y_{m}}\left(f_{l j}\right)=\partial_{x_{l}}\left(f_{m j}^{\prime}\right), \quad \partial_{y_{l}}\left(f_{m j}^{\prime}\right)=\partial_{y_{m}}\left(f_{l j}^{\prime}\right), \\
& \partial_{x_{l}}\left(g_{m j}\right)=\partial_{x_{m}}\left(g_{l j}\right), \quad \partial_{y_{m}}\left(g_{l j}\right)=\partial_{x_{l}}\left(g_{m j}^{\prime}\right), \quad \partial_{y_{l}}\left(g_{m j}^{\prime}\right)=\partial_{y_{m}}\left(g_{l_{j}}^{\prime}\right) \text {. }
\end{aligned}
$$

Since $U$ is simply connected, there are unique functions $\varphi_{j}$ and $\psi_{j}(1 \leqq j \leqq n)$ up to constants on $U$ such that

$$
\begin{array}{ll}
\partial_{x_{i}}\left(\varphi_{j}\right)=f_{i j}, & \partial_{y_{i}}\left(\varphi_{j}\right)=f_{i j}^{\prime}, \\
\partial_{x_{\mathrm{t}}}\left(\psi_{j}\right)=g_{i j}, & \partial_{y_{i}}\left(\psi_{j}\right)=g_{i j}^{\prime} .
\end{array}
$$

Here we may assume that all $\varphi_{i}$ and $\psi_{i}$ have no constant terms. Put

$$
Z^{\prime}=-\sum_{j=1}^{n}\left(\varphi_{j} \partial_{x_{j}}+\psi_{j} \partial_{y_{j}}\right)
$$

then we get

$$
\left[Z^{\prime}, \partial_{v_{i}}\right]=D\left(\partial_{v_{i}}\right) \quad(1 \leqq i \leqq 2 n) .
$$

Lemma 1. The vector field $Z^{\prime}$ defined above is hamiltonian modulo linear terms.

Note. The field $Z^{(2)}$ is determined as the component of $Z^{\prime}$ with coefficient functions of degree $\geqq 2$. The structure of the linear term of $Z^{\prime}, Z^{(1)}=Z^{\prime}-Z^{(2)}$, will be studied in $\S 3.3$.

Proof. Since $D\left(\partial_{v_{i}}\right)$ is hamiltonian for all $i$, the equalities (1) hold for $f_{i j}, g_{i j}$ and also for $f_{i j}^{\prime}, g_{i j}^{\prime}$. Hence for all $i, j, l$, we get

$$
\begin{gathered}
\partial_{x_{l}}\left(f_{i j}^{\prime}\right) \stackrel{(2)}{=} \partial_{y_{i}}\left(f_{l j}\right) \stackrel{(1)}{=} \partial_{y_{j}}\left(f_{l i}\right) \stackrel{(2)}{=} \partial_{x_{l}}\left(f_{j i}^{\prime}\right), \\
\partial_{y_{l}}\left(f_{i j}^{\prime}\right)=\partial_{y_{i}}\left(f_{l j}^{\prime}\right)=\partial_{y_{j}}\left(f_{l i}^{\prime}\right)=\partial_{y_{l}}\left(f_{j i}^{\prime}\right),
\end{gathered}
$$

and similarly,

$$
\partial_{x_{l}}\left(g_{i j}\right) \stackrel{(3)}{=} \partial_{\lambda_{i}}\left(g_{l j}\right) \stackrel{(1)}{=} \partial_{x_{j}}\left(g_{l i}\right) \stackrel{(3)}{=} \partial_{x_{l}}\left(g_{j i}\right)
$$




$$
\partial_{y_{l}}\left(g_{i j}\right)=\partial_{x_{i}}\left(g_{l j}^{\prime}\right)=\partial_{x_{j}}\left(g_{l i}^{\prime}\right)=\partial_{y_{l}}\left(g_{j i}\right)
$$

and further,

$$
\begin{gathered}
\partial_{x_{l}}\left(f_{i j}\right) \stackrel{(2)}{=} \partial_{x_{i}}\left(f_{l j}\right) \stackrel{(1)}{=}-\partial_{y_{j}}\left(g_{l i}\right) \stackrel{(3)}{=}-\partial_{x_{l}}\left(g_{j i}^{\prime}\right), \\
\partial_{y_{l}}\left(f_{i j}\right)=\partial_{x_{i}}\left(f_{l j}^{\prime}\right)=-\partial_{y_{j}}\left(g_{l i}^{\prime}\right)=-\partial_{y_{l}}\left(g_{j i}^{\prime}\right)
\end{gathered}
$$

From the above equalities, we have

$$
f_{i j}^{\prime} \equiv f_{j i}^{\prime}, \quad g_{i j} \equiv g_{j i}, \quad f_{i j}+g_{j i}^{\prime} \equiv 0,
$$

modulo constant terms for all $i, j$. This means that $Z^{\prime}$ satisfies, modulo linear terms, the condition (1) to be hamiltonian.

Q.E.D.

\subsection{The Structure of $Z^{(1)}$.}

We have just proved in the preceding paragraph that

$$
D\left(\partial_{v_{i}}\right)=\left[Z^{\prime}, \partial_{v_{i}}\right]=\left[Z^{(1)}, \partial_{v_{i}}\right]+\left[Z^{(2)}, \partial_{v_{i}}\right] \quad(1 \leqq i \leqq 2 n),
$$

and that $Z^{(2)}$ is hamiltonian. However $Z^{(1)}$ is not hamiltonian in general. Let us study the linear field $Z^{(1)}$ more in detail.

Put $D^{\prime}=D-\operatorname{ad} Z^{(2)}$, then we have

$$
\begin{aligned}
& D^{\prime}\left(\partial_{x_{i}}\right)=\sum_{k=1}^{n}\left(a_{i k} \partial_{x_{k}}+b_{i k} \partial_{y_{k}}\right), \\
& D^{\prime}\left(\partial_{y_{i}}\right)=\sum_{k=1}^{n}\left(c_{i k} \partial_{x_{k}}+d_{i k} \partial_{y_{k}}\right),
\end{aligned}
$$

where for all $i, k$,

$$
a_{i k}=f_{i k}(0), \quad b_{i k}=g_{i k}(0), \quad c_{i k}=f_{i k}^{\prime}(0), \quad d_{i k}=g_{i k}^{\prime}(0) .
$$

Then by (4)

$$
Z^{(1)}=-\sum_{k}\left\{\sum_{i}\left(a_{i k} x_{i}+c_{i k} y_{i}\right) \partial_{x_{k}}+\sum_{i}\left(b_{i k} x_{i}+d_{i k} y_{i}\right) \partial_{y_{k}}\right\}
$$

Let $X_{i j}, Y_{i j}$ and $Z_{i j}$ be the basis of the linear hamiltonian vector fields, given as

$$
X_{i j}=x_{i} \partial_{x_{j}}-y_{j} \partial_{y_{i}} \quad(1 \leqq i, j \leqq n),
$$




$$
\begin{array}{ll}
Y_{i j}=x_{i} \partial_{y_{j}}+x_{j} \partial_{y_{i}} & (1 \leqq i \leqq j \leqq n), \\
Z_{i j}=y_{i} \partial_{x_{j}}+y_{j} \partial_{x_{i}} & (1 \leqq i \leqq j \leqq n) .
\end{array}
$$

Define the functions $\bar{\alpha}_{i j k}$ etc. on $U$ for all $i, j, k$ by

$$
\begin{aligned}
& D^{\prime}\left(X_{i j}\right)=\sum_{k}\left(\bar{\alpha}_{i j k} \partial_{x_{k}}+\bar{\beta}_{i j k} \partial_{y_{k}}\right), \\
& D^{\prime}\left(Y_{i j}\right)=\sum_{k}\left(\bar{\gamma}_{i j k} \partial_{x_{k}}+\bar{\delta}_{i j k} \partial_{y_{k}}\right), \\
& D^{\prime}\left(Z_{i j}\right)=\sum_{k}\left(\bar{\xi}_{i j k} \partial_{x_{k}}+\bar{\eta}_{i j k} \partial_{y_{k}}\right)
\end{aligned}
$$

Then we have the following

Lemma 2. The functions $\bar{\alpha}_{i j k}, \bar{\beta}_{i j k}, \bar{\gamma}_{i j k}, \bar{\delta}_{i j k}, \bar{\xi}_{i j k}$ and $\bar{\eta}_{i j k}$ are of degree $\leqq 1$, whose linear terms are determined by the constants $a_{i k}$, $b_{i k}, c_{i k}$ and $d_{i k}$ in (5).

Proof. First, we have for all $i, l, m$,

$$
\left[\partial_{x_{i}}, X_{l m}\right]=\delta_{i l} \partial_{x_{m}},\left[\partial_{y_{i}}, X_{l m}\right]=-\delta_{i m} \partial_{y_{l}}
$$

where $\delta_{i j}$ is the Kronecker's delta. Applying $D^{\prime}$ to these equalities, we have

$$
\begin{aligned}
& \delta_{i l} \sum_{k}\left(a_{m k} \partial_{x_{k}}+b_{m k} \partial_{y_{k}}\right) \\
& \quad=\left[D^{\prime}\left(\partial_{x_{i}}\right), X_{l m}\right]+\left[\partial_{x_{i}}, D^{\prime}\left(X_{l m}\right)\right] \\
& \quad=a_{i l} \partial_{x_{m}}-b_{i m} \partial_{y_{l}}+\sum_{k}\left\{\partial_{x_{i}}\left(\bar{\alpha}_{l m k}\right) \partial_{x_{k}}+\partial_{x_{i}}\left(\bar{\beta}_{l m k}\right) \partial_{y_{k}}\right\} \\
& -\delta_{i m} \sum_{k}\left(c_{l k} \partial_{x_{k}}+d_{l k} \partial_{y_{k}}\right) \\
& \quad=c_{i l} \partial_{x_{m}}-d_{i m} \partial_{y_{l}}+\sum_{k}\left\{\partial_{y_{i}}\left(\bar{\alpha}_{l m k}\right) \partial_{x_{k}}+\partial_{y_{i}}\left(\bar{\beta}_{l m k}\right) \partial_{y_{k}}\right\}
\end{aligned}
$$

Compare the coefficients of $\partial_{x_{k}}$ and $\partial_{y_{k}}$, then we see that the derivatives of the first order in $x_{i}$ and $y_{i}$ of $\bar{\alpha}_{l m k}$ and $\bar{\beta}_{l m k}$ are constants determined by $a_{i j}, b_{i j}, c_{i j}$ and $d_{i j}$. Hence we have the assertion for $\bar{\alpha}_{i j k}$ and $\bar{\beta}_{i j k}$. 
By the same arguments, we have also the assertion for $\bar{\gamma}_{i j k}, \bar{\delta}_{i j k}$, $\bar{\xi}_{i j h}$ and $\bar{\eta}_{i j k}$.

Q.E.D.

Lemma 3. There are the following relations:

i) $\quad a_{i j}+d_{j i}=0 \quad(i \neq j)$,

ii) $\quad a_{i i}+d_{i i}=a_{j j}+d_{j j}$,

iii) $\quad b_{i j}=b_{j i} \quad$ for all $i, j$,

iv) $\quad c_{i j}=c_{j i} \quad$ for all $i, j$.

Proof. If $i \neq l$ in (6), we have

$$
0=a_{i l} \partial_{x_{m}}-b_{i m} \partial_{y_{l}}+\sum_{k}\left\{\partial_{x_{i}}\left(\bar{\alpha}_{l m h}\right) \partial_{x_{k}}+\partial_{x_{i}}\left(\bar{\beta}_{l m h}\right) \partial_{y_{k}}\right\},
$$

and hence

$$
\partial_{x_{l}}\left(\bar{\alpha}_{l m m}\right)=-a_{i l}, \quad \partial_{x_{i}}\left(\bar{\beta}_{l m l}\right)=b_{i m} \quad(i \neq l) .
$$

Put $i=l$ in (6), we have

$$
\begin{aligned}
& \sum_{k}\left(a_{m k} \partial_{x_{k}}+b_{m k} \partial_{y_{k}}\right) \\
& \quad=a_{i i} \partial_{x_{m}}-b_{i m} \partial_{y_{i}}+\sum_{k}\left\{\partial_{x_{i}}\left(\bar{\alpha}_{i m k}\right) \partial_{x_{k}}+\partial_{x_{i}}\left(\bar{\beta}_{i m k}\right) \partial_{y_{k}}\right\},
\end{aligned}
$$

which implies that

(9)

$$
\left\{\begin{array}{l}
\partial_{x_{i}}\left(\bar{\alpha}_{i m m}\right)=a_{m m}-a_{i i}, \\
\partial_{x_{i}}\left(\bar{\alpha}_{i m k}\right)=a_{m k} \quad(k \neq m), \\
\partial_{x_{i}}\left(\bar{\beta}_{i m k}\right)=b_{m k} \quad(k \neq i) .
\end{array}\right.
$$

Now if $i \neq m$ in (7), we have

$$
0=c_{i l} \partial_{x_{m}}-d_{i m} \partial_{y_{l}}+\sum_{k}\left\{\partial_{y_{i}}\left(\bar{\alpha}_{l m k}\right) \partial_{x_{k}}+\partial_{y_{i}}\left(\bar{\beta}_{l m k}\right) \partial_{y_{k}}\right\},
$$

and hence

$$
\partial_{y_{i}}\left(\bar{\alpha}_{l m m}\right)=-c_{i l}, \quad \partial_{y_{i}}\left(\bar{\beta}_{l m l}\right)=d_{i m} \quad(i \neq m) .
$$


Similarly for $i=m$, we get

$$
\begin{aligned}
& -\sum_{k}\left(c_{l k} \partial_{x_{k}}+d_{l k} \partial_{y_{k}}\right) \\
= & c_{i l} \partial_{x_{i}}-d_{i i} \partial_{y_{l}}+\sum_{k}\left\{\partial_{y_{i}}\left(\bar{\alpha}_{l i k}\right) \partial_{x_{k}}+\partial_{y_{i}}\left(\bar{\beta}_{l i k}\right) \partial_{y_{k}}\right\},
\end{aligned}
$$

which implies that

$$
\left\{\begin{array}{l}
\partial_{y_{i}}\left(\bar{\beta}_{l i l}\right)=d_{i i}-d_{l l}, \\
\partial_{y_{i}}\left(\bar{\alpha}_{l i k}\right)=-c_{l k} \quad(k \neq i) .
\end{array}\right.
$$

Let us take into consideration the condition (1) that $D^{\prime}\left(X_{i j}\right)$ 's are hamiltonian, then we have from (9) and (10), for $m \neq k$,

$$
0=\partial_{x_{i}}\left(\bar{\alpha}_{i m k}\right)+\partial_{y_{k}}\left(\bar{\beta}_{i m i}\right)=a_{m k}+d_{k m}
$$

which means i).

Also we obtain from (9) and (11),

$$
0=\partial_{x_{i}}\left(\bar{\alpha}_{i j j}\right)+\partial_{y_{j}}\left(\bar{\beta}_{i j i}\right)=a_{j j}-a_{i i}+d_{j j}-d_{i i}
$$

which means ii); and from (8) and (9), for $i \neq l$,

$$
0=\partial_{x_{i}}\left(\bar{\beta}_{l m l}\right)-\partial_{x_{l}}\left(\bar{\beta}_{l m i}\right)=b_{i m}-b_{m i},
$$

which means iii); and from (10) and (11), for $i \neq m$,

$$
0=\partial_{y_{i}}\left(\bar{\alpha}_{l m m}\right)-\partial_{y_{m}}\left(\bar{\alpha}_{l m i}\right)=-c_{i l}+c_{l i}
$$

which means iv).

Q.E.D.

Remark 1. There is no relation besides i) iv) among $a_{i j}, b_{i j}, c_{i j}$ and $d_{i j}$, which comes from the condition that $D^{\prime}(V)$ is hamiltonian, where $V$ is any one of $X_{i j}, Y_{i j}$ and $Z_{i j}$. Further more there holds

$$
D^{\prime}(V) \equiv\left[Z^{(1)}, V\right]
$$

modulo constant terms, where $V$ is as above.

Now we can describe the structure of $Z^{(1)}$. 
Lemma 4. The vector field $Z^{(1)}$ is uniquely expressed as

$$
Z^{(1)}=Z_{1}^{(1)}+Z_{2}
$$

where $Z_{1}^{(1)}$ is hamiltonian, and for some constant $c$,

$$
Z_{2}=c \sum_{i=1}^{n}\left(x_{i} \partial_{x_{i}}+y_{i} \partial_{y_{i}}\right)
$$

Proof. Put

$$
Z^{(1)}=-\sum_{i=1}^{n}\left\{f_{i}(x, y) \partial_{x_{i}}+g_{i}(x, y) \partial_{y_{i}}\right\}
$$

then from (5), we have for $i=1, \ldots, n$,

$$
\begin{aligned}
& f_{i}(x, y)=\sum_{j=1}^{n}\left(a_{j i} x_{j}+c_{j i} y_{j}\right), \\
& g_{i}(x, y)=\sum_{j=1}^{n}\left(b_{j i} x_{j}+d_{j i} y_{j}\right) .
\end{aligned}
$$

Put $c=2^{-1}\left(a_{i i}+d_{i i}\right)$, which is independent of $i$ by Lemma 3 ii), and put

$$
Z_{2}=c \sum_{i}\left(x_{i} \partial_{x_{i}}+y_{i} \partial_{y_{i}}\right)
$$

Then, the remaining term $Z_{1}^{(1)}=Z^{(1)}-Z_{2}$ is hamiltonian by the equalities i) iv) in Lemma 3. One can easily see that the decomposition $Z^{(1)}=$ $Z_{1}^{(1)}+Z_{2}$ is unique, as far as $Z_{1}^{(1)}$ is hamiltonian and $Z_{2}$ is a scalar multiple of $\sum_{i}\left(x_{i} \partial_{x_{i}}+y_{i} \partial_{y_{i}}\right)$.

Q.E.D.

\subsection{Determination of $Z^{(0)}$.}

Let the derivation $D^{\prime \prime}$ be $D^{\prime \prime}=D-\operatorname{ad} Z^{\prime}$, then by $\S 3.2$. we have

$$
D^{\prime \prime}\left(\partial_{v_{i}}\right)=0 \quad(1 \leqq i \leqq 2 n) .
$$

Let $\alpha_{i j k}, \beta_{i j k}, \gamma_{i j k}, \delta_{i j k}, \xi_{i j k}$ and $\eta_{i j k}$ be the constant terms of $\bar{\alpha}_{i j k}$, $\bar{\beta}_{i j k}, \bar{\gamma}_{i j k}, \bar{\delta}_{i j k}, \bar{\xi}_{i j k}$ and $\bar{\eta}_{i j k}$ respectively, then, by Lemma 2, we have for all $i, j, k$,

$$
D^{\prime \prime}\left(X_{i j}\right)=\sum_{k}\left(\alpha_{i j k} \partial_{\lambda_{k}}+\beta_{i j k} \partial_{y_{k}}\right)
$$




$$
\begin{aligned}
& D^{\prime \prime}\left(Y_{i j}\right)=\sum_{k}\left(\gamma_{i j h} \partial_{x_{k}}+\delta_{i j k} \partial_{y_{k}}\right), \\
& D^{\prime \prime}\left(Z_{i j}\right)=\sum_{k}\left(\xi_{i j k} \partial_{x_{k}}+\eta_{i j k} \partial_{y_{k}}\right) .
\end{aligned}
$$

Moreover $\alpha_{i j k}$ etc. are expressed more simply as follows.

\section{Lemma 5.}
i) $\quad D^{\prime \prime}\left(X_{i j}\right)=\alpha_{i} \partial_{x_{j}}+\beta_{j} \partial_{y_{i}}$,
ii) $\quad D^{\prime \prime}\left(Y_{i j}\right)=\alpha_{i} \partial_{y_{j}}+\alpha_{j} \partial_{y_{i}}$,
iii) $\quad D^{\prime \prime}\left(Z_{i j}\right)=-\beta_{i} \partial_{x_{j}}-\beta_{j} \partial_{x_{i}}$,

where $\alpha_{i}=\alpha_{i i i}, \beta_{i}=\beta_{i i i}$.

Proof. Applying $D^{\prime \prime}$ to the both sides of the equality

$$
\left[X_{i j}, X_{l m}\right]=\delta_{j l} X_{i m}-\delta_{i m} X_{l j}
$$

then we get

$$
\begin{gathered}
\delta_{j l} \sum_{k}\left(\alpha_{i m k} \partial_{x_{h}}+\beta_{i m k} \partial_{y_{h}}\right)-\delta_{i m} \sum_{k}\left(\alpha_{l j k} \partial_{x_{k}}+\beta_{l j h} \partial_{y_{k}}\right) \\
=\left[\sum_{k}\left(\alpha_{i j k} \partial_{x_{k}}+\beta_{i j k} \partial_{y_{h}}\right), x_{l} \partial_{\lambda_{m}}-y_{m} \partial_{y_{l}}\right] \\
+\left[x_{i} \partial_{x_{j}}-y_{J} \partial_{y_{i}}, \sum_{k}\left(\alpha_{l m k} \partial_{\lambda_{k}}+\beta_{l m k} \partial_{y_{h}}\right)\right] \\
=\alpha_{i j l} \partial_{x_{m}}-\beta_{i j m} \partial_{y_{l}}-\alpha_{l m i} \partial_{x_{j}}+\beta_{l m j} \partial_{y_{i}} .
\end{gathered}
$$

If $j \neq l$ and $i \neq m$ in (12), we have

$$
0=\alpha_{i j l} \partial_{x_{m}}-\beta_{i j m} \partial_{y_{l}}-\alpha_{l m i} \partial_{x_{j}}+\beta_{l m j} \partial_{y_{i}},
$$

which implies that for $j \neq m$,

$$
\alpha_{i j l}=0 \quad(j \neq l),
$$

and for $i \neq l$,

$$
\beta_{i j m}=0 \quad(i \neq m) .
$$


Then if $j=l$ and $i \neq m$ in (12), we have

$$
\alpha_{i m m} \partial_{x_{m}}+\beta_{i m i} \partial_{y_{i}}=\alpha_{i l l} \partial_{x_{m}}+\beta_{l m l} \partial_{y_{i}},
$$

and hence

$$
\left\{\begin{array}{l}
\alpha_{i m m}=\alpha_{i l l}=\alpha_{i i i}, \\
\beta_{i m i}=\beta_{l m l}=\beta_{m m m} .
\end{array}\right.
$$

Put $\alpha_{i}=\alpha_{i i i}, \beta_{i}=\beta_{i i i}$, then

$$
D^{\prime \prime}\left(X_{i j}\right)=\alpha_{i j j} \partial_{x_{j}}+\beta_{i j i} \partial_{y_{i}}=\alpha_{i} \partial_{x_{j}}+\beta_{j} \partial_{y_{i}},
$$

which is the cquality i).

Applying $D^{\prime \prime}$ also to the both sides of the equality

$$
\left[X_{i j}, Y_{l m}\right]=\delta_{j l} Y_{i m}+\delta_{j m} Y_{i l}
$$

we get

$$
\begin{gathered}
\delta_{j l} \sum_{k}\left(\gamma_{i m k} \partial_{x_{k}}+\delta_{i m k} \partial_{y_{k}}\right)+\delta_{j m} \sum_{k}\left(\gamma_{i l k} \partial_{x_{k}}+\delta_{i l k} \partial_{y_{k}}\right) \\
=\left[\alpha_{i} \partial_{x_{j}}+\beta_{j} \partial_{y_{i}}, x_{l} \partial_{y_{m}}+x_{m} \partial_{y_{l}}\right] \\
+\left[x_{i} \partial_{x_{j}}-y_{J} \partial_{y_{l}}, \sum_{k}\left(\gamma_{l m k} \partial_{x_{k}}+\delta_{l m h} \partial_{y_{h}}\right)\right] \\
=\delta_{j l} \alpha_{l} \partial_{y_{m}}+\delta_{j m} \alpha_{i} \partial_{y_{l}}-\gamma_{l m i} \partial_{x_{J}}+\delta_{l m,} \partial_{y_{i}} .
\end{gathered}
$$

If $j \neq l$ and $j \neq m$ in (13), we obtain

$$
0=-\gamma_{l m i} \partial_{x_{j}}+\delta_{l m j} \partial_{y_{i}}
$$

and hence for all $l, m, i$,

$$
\begin{aligned}
& \gamma_{l m i}=0, \\
& \delta_{l m j}=0 \quad(j \neq m, l) .
\end{aligned}
$$

Then put $i \neq j=m=l$ in (13), we obtain

$$
2 \sum_{k} \delta_{i j k} \partial_{y_{k}}=2 \alpha_{i} \partial_{y_{j}}+\delta_{j j j} \partial_{y_{i}}
$$

which implies, by the symmetry of $\delta_{i j k}$ in $i$ and $j$, that 


$$
\begin{aligned}
& \alpha_{i}=\delta_{i j j}=\delta_{j i j}, \\
& \delta_{j j j}=2 \delta_{i j i}=2 \alpha_{j}
\end{aligned}
$$

Hence we have

$$
D^{\prime \prime}\left(Y_{i j}\right)=\delta_{i j j} \partial_{y_{j}}+\delta_{i j i} \partial_{y_{i}}=\alpha_{i} \partial_{y_{j}}+\alpha_{j} \partial_{y_{i}}
$$

for all $i, j$, which is the equality ii).

Apply $D^{\prime \prime}$ to the both sides of the equality

$$
\left[X_{i j}, Z_{l m}\right]=-\delta_{i l} Z_{j m}-\delta_{i m} Z_{j l}
$$

Then we get the equality iii) by the same arguments as for ii). Q.E.D.

Thus we have the hamiltonian vector field $Z^{(0)}$, given as

$$
Z^{(0)}=\sum_{i=1}^{n}\left(\alpha_{i} \partial_{x_{i}}-\beta_{i} \partial_{y_{i}}\right)
$$

with constants $\alpha_{i}, \beta_{i}$ in Lemma 5, such that for any linear hamiltonian vector field $V$

$$
\left[Z^{(0)}, V\right]=D^{\prime \prime}(V)
$$

However this condition determines $Z^{(0)}$ by the following lemma.

Lemma 6. Let $V$ be a constant (hamiltonian) vector field with $\left[V, X_{i j}\right]=0$ for all $i, j$, then we have that $V=0$.

Proof. Put

$$
V=\sum_{i=1}^{n}\left(a_{i} \partial_{x_{i}}+b_{i} \partial_{y_{i}}\right) \quad\left(a_{i}, b_{i} \in R\right)
$$

then we have

$$
0=\left[V, X_{i j}\right]=a_{i} \partial_{x_{j}}-b_{j} \partial_{y_{i}},
$$

and hence $a_{i}=b_{i}=0$, for all $i$.

Q.E.D.

3.5. Thus we know the vector field $Z$

$$
Z=Z^{(0)}+Z^{(1)}+Z^{(2)},
$$


as (4), (5) and (14) such that $D(V)=[Z, V]$ for all $V$ with coefficient functions of degree $\leqq 1$. Then we must show that $D$ is $\operatorname{ad} Z$ for all hamiltonian vector fields on $U$. This is established by the following

Lemma 7. Assume that a derivation $D$ vanishes at any $X$ such that the coefficient functions of $X$ are of degree $\leqq 1$. Then $D$ is identically zero on $\mathfrak{H}_{\omega}(U)$.

To prove this, we use the following.

Lemma 8. Under the assumption of Lemma $7, D(X)=0$, if all coefficient functions of $X$ are of degree 2.

The proof of this lemma will be given in $\S 4$.

Proof of Lemma 7. Let $X \in \mathfrak{U}_{\omega}(U)$, then we can show that $D(X)(p)$ $=0$ for any point $p \in U$. In fact, there is a decomposition of $X$ at $p$, $X=X_{1}+X_{2}$ such that the coefficient functions of $X_{1}$ are polynomials of degree $\leqq 2$, and $j^{2}\left(X_{2}\right)(p)=0$. Then by Lemma 8 and Proposition 4 , we have

$$
D(X)(p)=D\left(X_{1}\right)(p)+D\left(X_{2}\right)(p)=0
$$

Q.E.D.

\section{§4. Relations to the Formal Lie Algebras}

4.1. It is known by $\mathrm{T}$. Morimoto [9] that the derivation algebra of the following irreducible transitive Lie algebra (TLA) $g$ of infinite type:

$$
\mathfrak{g}=V^{2 n}+\mathfrak{s p}(V)+\mathfrak{s p}(V)^{(1)}+\cdots+\mathfrak{s p}(V)^{(p)}+\cdots,
$$

(for definition, see [5] for example) has the subspace of outer derivations, of dimension 1. In other words, $H^{1}\left(\mathfrak{A}_{\omega}(n) ; \mathfrak{A}_{\omega}(n)\right) \cong \mathbb{R}$, where $\mathfrak{A}_{\omega}(n)$ is the Lie algebra of formal hamiltonian vector fields on $\mathbb{R}^{2 n}$ at the origin (for definition, see [3] for example). By some techniques used to prove the above formal theorem, we have another approach to the determination of $Z^{(0)}$, and a proof of Lemma 8 .

The constant hamiltonian vector fields form a Euclidean vector 
space (abelian Lie algebra) $V_{0}$ of dimension $2 n$, and the linear hamiltonian fields form a vector space $V_{1} \cong \mathfrak{s p}\left(V_{0}\right)$, with the natural structure of Lie algebra. Before Lemma 5 , we have already proved that $D^{\prime \prime}\left(V_{1}\right) \subset$ $V_{0}$. The natural representation of $\mathfrak{s p}(2 n ; R)$ on $\mathbb{R}^{2 n}$ is irreducible, and is given in terms of vector fields as $X\left(\partial_{v}\right)=\left[\partial_{v}, X\right]$, where $X \in V_{1} \cong$ $\mathfrak{s p}(2 n ; \mathbb{R})$ and $\partial_{v} \in V_{0} \cong \mathbb{R}^{2 n}$.

Thus the linear map (derivation) $D^{\prime \prime}$ from $V_{1}$ to $V_{0}$ is a 1-cocycle of $\mathfrak{s p}(2 n ; \mathbb{R})$ with coefficients in the above representation. Apply to $D^{\prime \prime}$ the fundamental vanishing theorem for nontrivial irreducible representations of (finite dimensional) semi-simple Lie algebras (cf. [1]). Then we get a unique vector $v_{0} \in V_{0}$ such that

$$
D^{\prime \prime}(X)=\left(d v_{0}\right)(X)=X\left(v_{0}\right)=\left[Z^{(0)}, X\right] \quad\left(X \in V_{1}\right) .
$$

Here, $Z^{(0)}$ is the vector field corresponding to the vector $v_{0}$, and expressed according to the formula in [1] as follows:

$$
\begin{aligned}
Z^{(0)}= & (2 n+1)^{-1}(2 n+2)^{-2}\left\{\sum_{i, j}\left[X_{i j}, D^{\prime \prime}\left(X_{j i}\right)\right]+\sum_{i<j}\left(\left[Y_{i j}, D^{\prime \prime}\left(Z_{i j}\right)\right]\right.\right. \\
& \left.\left.+\left[Z_{i j}, D^{\prime \prime}\left(Y_{i j}\right)\right]\right)+4^{-1} \sum_{i}\left(\left[Y_{i i}, D^{\prime \prime}\left(Z_{i i}\right)\right]+\left[Z_{i i}, D^{\prime \prime}\left(Y_{i i}\right)\right]\right)\right\} .
\end{aligned}
$$

(It is not easy to obtain the explicit formula (14) of $Z^{(0)}$ from the above expression of it.)

\subsection{Proof of Lemma 8.}

The hamiltonian vector fields of homogeneous degree 2, also form a vector space $V_{2} \cong \mathfrak{s p}\left(V_{0}\right)^{(1)}$, the first prolongation of $V_{1}=\mathfrak{s p}\left(V_{0}\right)$. The natural representation of $V_{1}$ on $V_{2}$ is given in terms of vector fields as $X(Y)=[Y, X]$ for $X \in V_{1}$ and $Y \in V_{2}$. Then it is known by $H$. Weyl [8] that this representation is irreducible.

As in the proof of Lemma 2, we have $D\left(V_{2}\right) \subset V_{0}$. From the assumption of Lemma 8 , we see that $D([X, Y])=[X, D(Y)]$ for $X \in V_{1}$ and $Y \in V_{2}$. Then the following diagram is commutative:

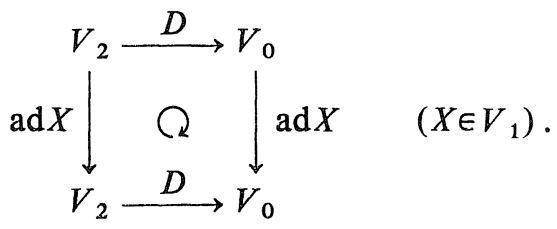


This implies that $\operatorname{ker} D$ is stable under $\operatorname{ad}\left(V_{1}\right)$-actions. Since $\operatorname{ker} D \neq\{0\}$ clearly, it follows from the irreducibility of the representation that $\operatorname{ker} D=V_{2}$, that is $D=0$ on $V_{2}$.

Q.E.D.

Remark 1. This proof is simple and short, but is based upon Weyl's work [8]. We have another proof by elementary calculations. Let us sketch it here.

Take a basis $X_{i j k}, Y_{i j k}, Z_{i j k}$ and $W_{i j k}$ of $V_{2}$, as

$$
\begin{aligned}
& X_{i j k}=x_{i} x_{j} \partial_{x_{k}}-x_{j} y_{k} \partial_{y}-x_{i} y_{k} \partial_{y_{j}}, \\
& Y_{i j k}=x_{k} y_{i} \partial_{x_{j}}+x_{k} y_{j} \partial_{x_{i}}-y_{i} y_{j} \partial_{y_{k}}, \\
& Z_{i j k}=y_{i} y_{j} \partial_{x_{k}}+y_{j} y_{k} \partial_{x_{i}}+y_{k} y_{i} \partial_{x_{j}}, \\
& W_{i j k}=x_{i} x_{j} \partial_{y_{k}}+x_{j} x_{k} \partial_{y_{i}}+x_{k} x_{i} \partial_{y_{j}}
\end{aligned}
$$

Define the functions $a_{i j k l}$ etc. on $U$ by

$$
\begin{aligned}
& D\left(X_{i j k}\right)=\sum_{l}\left(a_{i j k l} \partial_{x_{l}}+a_{i j k l}^{\prime} \partial_{y_{l}}\right), \\
& D\left(Y_{i j k}\right)=\sum_{l}\left(b_{i j k l} \partial_{x_{l}}+b_{i j k l}^{\prime} \partial_{y_{l}}\right), \\
& D\left(Z_{i j k}\right)=\sum_{l}\left(c_{i j k l} \partial_{x_{l}}+c_{i j k l}^{\prime} \partial_{y_{l}}\right), \\
& D\left(W_{i j k}\right)=\sum_{l}\left(d_{i j k l} \partial_{x_{l}}+d_{i j k l}^{\prime} \partial_{y_{l}}\right) .
\end{aligned}
$$

Then all these functions are constants as in the proof of Lemma 2. Moreover these constants are zero. In fact, firstly we obtain that $a_{i j k l}=$ $d_{i j k l}=d_{i j k l}^{\prime}=0$ for all $i, j, k, l$, by applying $D$ to the both sides of the equality

$$
\left[X_{i j k}, Y_{m n}\right]=\delta_{m k} W_{i j k}+\delta_{n k} W_{i j n},
$$

and by the symmetry of $d_{i j k l}^{\prime}$ in $i, j, k$.

Secondly we obtain that $b_{i j k l}^{\prime}=c_{i j k l}=c_{i j k l}^{\prime}=0$, by applying $D$ to the equality

$$
\left[Z_{m n}, Y_{i j k}\right]=\delta_{n k} Z_{i j m}+\delta_{m k} Z_{i j n},
$$

and by the symmetry of $c_{i j k l}$ in $i, j, k$. 
Finally we get that $a_{i j k l}^{\prime}=b_{i j k l}=0$, by applying $D$ to the equality

$$
\left[Z_{m n}, X_{i j k}\right]=\delta_{n i} Y_{m k j}+\delta_{n j} Y_{m k i}+\delta_{m i} Y_{n k J}+\delta_{m j} Y_{n k i},
$$

and by the symmetry of $b_{i j k l}$ in $i, j$.

Thus we have that $D=0$ on $V_{2}$.

\section{§5. The Cohomology $\boldsymbol{H}^{1}\left(\mathfrak{P}_{\omega}(M) ; \mathfrak{I}_{\omega}(M)\right)$}

5.1. In the preceding two sections, we proved Theorem 5, a local theorem. The following one follows immediately from it.

Theorem 5'. $H^{1}\left(\mathfrak{H}_{\omega}(U) ; \mathfrak{A}_{\omega}(U)\right) \cong \mathbf{R}$

Now we will give a global theorem on $M$. Before that, we show a global version corresponding to Theorem 5 .

Proposition 6. Let $(M, \omega)$ be a symplectic manifold, and $D$ a derivation of hamiltonian vector fields $\mathfrak{A}_{\omega}(M)$ on $M$. Then there exists a vector field $Z$ on $M$ such that

$$
D(X)=[Z, X] \quad \text { for all } X \in \mathfrak{A}_{\omega}(M) .
$$

Proof. Take an atlas $\left\{U_{l}, \varphi_{i}: U_{i} \rightarrow \mathbf{R}^{2 n}\right\}$ of $M$ such that each $U_{l}$ is a simply connected domain. Then, by Theorem $5 \mathrm{i}$ ) and Remark 1 in $\S 2$, we have on each $U_{i}$ a vector field $Z_{U_{i}}$ such that $D_{U_{i}}(X)=\left[Z_{U_{i}}, X\right]$

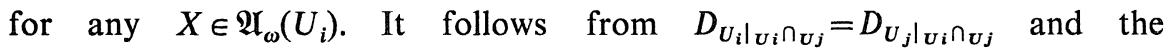

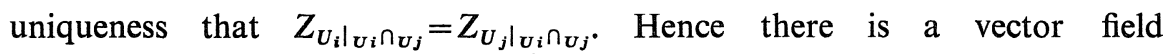
$Z \in \mathfrak{A}(M)$ such that $Z_{\mid U_{\imath}}=Z_{U_{i}}$ for each $U_{i}$ and that $D(X)=[Z, X]$ for any $X \in \mathfrak{A}_{\omega}(M)$.

Q.E.D.

Let $U$ be a simply connected domain, and $\left(x_{1}, \ldots, x_{n}, y_{1}, \ldots, y_{n}\right)$ a symplectic coordinate system such that $\omega_{\mid U}=\Sigma d x_{i} d y_{i}$. Then, by Theorem 5 ii), the above vector field $Z$ is represented as $Z=Z_{1 U}+Z_{2 U}$ on $U$, where $Z_{1 U} \in \mathfrak{U}_{\omega}(U)$ and $Z_{2 U}=c \sum_{i}\left(x_{i} \partial_{x_{i}}+y_{i} \partial_{y_{i}}\right)$ for some constant $c$. Then we have the following

Proposition 7. If $M$ is connected, the constant $c$ is independent of the choice of $U$ and $\left(x_{1}, \ldots, x_{n}, y_{1}, \ldots, y_{n}\right)$. 
Proof. Since $M$ is connected, it is sufficient to show that the constant $c$ is invariant under any symplectic coordinate transformations of $U$.

CASE 1. (Translations). Let new coordinates $\left(\bar{x}_{i}, \bar{y}_{i}\right)$ be

$$
\bar{x}_{i}=x_{\imath}-a_{i}, \quad \bar{y}_{i}=y_{i}-b_{i} \quad(1 \leqq i \leqq n)
$$

where $a_{i}, b_{i}$ are real constants. Then

$$
\left(\sum_{i} x_{i} \partial_{x_{i}}+y_{i} \partial_{y_{i}}\right)=\sum_{i}\left(\bar{x}_{i} \partial_{\bar{x}_{\iota}}+\bar{y}_{\imath} \partial_{\bar{y}_{i}}\right)+\sum_{i}\left(a_{i} \partial_{\bar{x}_{i}}+b_{i} \partial_{\bar{y}_{i}}\right)
$$

Since any constant vector field is hamiltonian, the constant $c$ is left invariant.

CASE 2. (Linear transformations). We prove that the constant $c$ is left invariant under any general linear transformation, not necessarily symplectic.

Take an element $g=\left(g_{i j}\right)$ in $G L(2 n ; \mathbf{R})$ and put

$$
\bar{v}_{i}=\sum_{j=1}^{2 n} g_{i j} v_{J} \quad(1 \leqq i \leqq 2 n)
$$

Then

$$
\partial_{v_{i}}=\sum_{j} \partial_{v_{i}}\left(\bar{v}_{j}\right) \partial_{\bar{v}_{j}}=\sum_{j} g_{j i} \partial_{\bar{v}_{j}}
$$

and hence

$$
\begin{aligned}
\sum_{i} v_{i} \partial_{v_{i}} & =\sum_{i}\left(\sum_{l}\left(g^{-1}\right)_{i l} \bar{v}_{l}\right)\left(\sum_{j} g_{j i} \partial_{\bar{v}_{j}}\right) \\
& =\sum_{j, l} \delta_{j l} \bar{v}_{l} \partial_{\bar{v}_{j}}=\sum_{j} \bar{v}_{j} \partial_{\bar{v}_{j}}
\end{aligned}
$$

CASE 3 (General case). We may assume by Case 1 that a symplectic coordinate transformation $\varphi$ has no constant terms. Then the inverse $\varphi^{-1}$ has also no constant terms. The vector fields $Z$ and $Z_{1}$ are transformed into hamiltonian ones by means of $\varphi$, and the linear term of the expression $\sum_{i}\left(x_{i} \partial_{x_{i}}+y_{i} \partial_{y_{i}}\right)$ in terms of new coordinates depends only on the linear parts of the transformations $\varphi$ and $\varphi^{-1}$. Hence we see by Casc 2 that the constant $c$ is invariant under $\varphi$, because the higher terms sum up to hamiltonian vector fields by Theorem $5 . \quad$ Q.E.D. 
Corollary. Let $Z$ and $c$ be as above, then $L_{Z} \omega=2 c \omega$ on $M$.

Proof. We see that for any $U$,

$$
L_{Z} \omega=L_{Z_{2 U}} \omega=2 c \omega \quad \text { on } U
$$

Q.E.D.

5.2. Now we can prove our main results.

Theorem 8. Let $(M, \omega)$ be a connected symplectic manifold. Then the first cohomology of the Lie algebra $\mathfrak{U}_{\omega}(M)$ with coefficients in its adjoint representation is of dimension 1 or 0 , that is,

$$
H^{1}\left(\mathfrak{H}_{\omega}(M) ; \mathfrak{P}_{\omega}(M)\right) \cong \mathbf{R} \text { or } 0 \text {. }
$$

Proof. We can define the homomorphism

$$
\phi: \mathfrak{D}_{\omega}(M) \longrightarrow \mathbf{R}
$$

which assigns to a derivation $D \in \mathfrak{D}_{\omega}(M)$ a constant $c$ by Proposition 7. Let us show that $\operatorname{ker} \phi=\mathfrak{D}_{\omega}^{i}(M)$. This means that

$$
H^{1}\left(\mathfrak{H}_{\omega}(M) ; \mathfrak{A}_{\omega}(M)\right) \cong \mathfrak{D}_{\omega} / \mathfrak{D}_{\omega}^{i} \cong \mathbf{R} \text { or } 0 \text { 。 }
$$

Let $D$ and $D^{\prime}$ be two derivations such that $\phi(D)=\phi\left(D^{\prime}\right)=c$, and put $\bar{D}=D-D^{\prime}$, then $\phi(\bar{D})=0$. By Remark 1 in $\S 2$ and Theorem $5, \bar{D}$ is inner on any sufficiently small simply connected domain $U$, that is, there exists a unique hamiltonian vector field $Z_{U}$ such that $\bar{D}_{\mid U}=\operatorname{ad}\left(Z_{U}\right)$. From the uniqueness in Theorem 5 , and by the same arguments in the proof of Proposition 6, there exists a unique vector field $Z$ whose restriction $Z_{\mid U}$ is equal to $Z_{U}$ for each such $U$. Clearly $Z$ is hamiltonian, and $\bar{D}(X)=[Z, X]$ for all $X \in \mathfrak{A}_{\omega}(M)$. Hence we have that ker $\phi$ $\subset \mathfrak{D}_{\omega}^{i}(M)$.

On the other hand, the converse inclusion: $\mathfrak{D}_{\omega}^{i}(M) \subset \operatorname{ker} \phi$, is clear.

Q.E.D.

Theorem 9. Assume that the symplectic from $\omega$ of $M$ is exact, or there exists a 1-form $\theta$ on $M$ such that $d \theta=\omega$. Then

$$
H^{1}\left(\mathfrak{A}_{\omega}(M) ; \mathfrak{A}_{\omega}(M)\right) \cong \mathbf{R}
$$


Proof. Let $W$ be a vector field corresponding to $\theta$ with respect to $\omega$, that is, $i_{W} \omega=\theta$. Then

$$
\omega=d 0=d i_{W} \omega=L_{W} \omega
$$

and hence $W$ is not hamiltonian. On the other hand,

$$
L_{[W, X]} \omega=L_{W} L_{X} \omega-L_{X} L_{W} \omega=-L_{X} \omega=0
$$

for all $X \in \mathfrak{A}_{\omega}(M)$, then $[W, X] \in \mathfrak{A}_{\omega}(M)$. Therefore ad $W$ is an outer derivation of $\mathfrak{S}_{\omega}(M)$.

Q.E.D.

Theorem 10. Assume that the symplectic form $\omega$ of $M$ is not exact. Then

$$
H^{1}\left(\mathfrak{A}_{\omega}(M) ; \mathfrak{H}_{\omega}(M)\right)=0
$$

Proof. Let $D$ be a derivation of $\mathfrak{A}_{\omega}(M)$. Then by Proposition 6, there is a unique vector field $Z \in \mathfrak{A}(M)$ such that $D=\operatorname{ad} Z$, and by Corollary of Proposition $7, L_{Z} \omega=c \omega$ for some constant $c$. Assume that $c \neq 0$, then $\omega=c^{-1} d\left(i_{Z} \omega\right)$, or $\omega$ is exact. Hence $c=0$, that is, $Z$ is hamiltonian. Thus all derivations of $\mathfrak{H}_{\omega}(M)$ are inner. Q.E.D.

Summarizing these results, we get the following Main Theorem.

Main Theorem. Let $\left(M^{2 n}, \omega\right)$ be a connected symplectic manifold, then

$$
\operatorname{dim} H^{1}\left(\mathfrak{A}_{\omega}(M) ; \mathfrak{H}_{\omega}(M)\right)=1 \text { or } 0
$$

Moreover, $H^{1}\left(\mathfrak{F}_{\omega}(M) ; \mathfrak{A}_{\omega}(M)\right) \cong \mathbb{R}$ if and only if the symplectic form $\omega$ is exact.

Remark 1. Let $M$ be a manifold attached with a volume form $\tau$ or a contact form $\mathfrak{c}$. Then, in stead of $\mathfrak{A}_{\omega}(M)$, we have a natural subalgebra $\mathfrak{U}_{\tau}(M)$ or $\mathfrak{P}_{\mathfrak{c}}(M)$ consisting of vector fields which preserves $\tau$ or $c$ respectively. It is interesting to obtain the analogous results for these subalgebras. If $n=1$, the above Main Theorem gives the result for $\mathfrak{A}_{\tau}(M)$ where $M$ is a 2-dimensional smooth manifold. 
Remark 2. The condition of continuity is absent in the definition of cochains of $\mathfrak{A}_{\omega}(M)$ with coefficients in its adjoint representation, but from the above results all cocycles are continuous.

\section{References}

[1] Chevalley, C. and Eilenberg, S., Cohomology theory of Lie groups and Lie algebras, Trans. A. M. S. 63 (1948), 85-124.

[2 ] Gel'fand, I. M. and Fuks, D. B., Cohomologies of Lie algebras of vector fields with nontrivial coefficients, Func. Anal. and its Appl. 4-3 (1970), 10-25 (in Russian).

[3] Gel'fand, I. M., Kalinin, D. I. and Fuks, D. B., On cohomologies of Lie algebras of Hamiltonian vector fields, ibid. 6-3 (1972), 25-29 (in Russian).

[4] Losik, M. V., On the cohomologies of infinite-dimensional Lie algebras of vector fields, ibid. $\mathbb{4}-2$ (1970), 43-53 (in Russian).

[5] Morimoto, T. and Tanaka, N., The classification of the real primitive infinite Lie algebras, J. Math. of Kyoto Univ. 10 (1970), 207-243.

[6] Rozenfel'd, B. I., Cohomologies of some infinite dimensional Lie algebras, Func. Anal. and its Appl. 5-4 (1971), 84-85 (in Russian).

[7] Takens, F., Derivations of vector fields, Comp. Math. 26 (1973), 151-158.

[8] Weyl, H., The Classical Groups; their invariants and representations. Princeton Univ. Press, 2nd ed., (1946).

[9] Morimoto, T., Derivation algebras of the classical infinite Lie algebras (unpublished).

Added in proof. The author was informed of the following work by the referee;

Avez, A., Lichnerowicz, A. and Diaz-Miranda, A., Sur l'algèbre des automorphismes infinitesimaux d'une variété symplectique, J. Differential Geometry 9 (1974), 1-40.

This paper contains essentially the same result as our main theorem, but the method of its proof is different from ours. 